\title{
Combination of saponification and dispersive liquid-liquid microextraction for the determination of tocopherols and tocotrienols in cereals by reversed-phase high- performance liquid chromatography
}

\begin{abstract}
A simple sample preparation technique coupled with reversed-phase high-performance liquid chromatography was developed for the determination of tocopherols and tocotrienols in cereals. The sample preparation procedure involved a small-scale hydrolysis of $0.5 \mathrm{~g}$ cereal sample by saponification, followed by the extraction and concentration of tocopherols and tocotrienols from saponified extract using dispersive liquid-liquid microextraction (DLLME). Parameters affecting the DLLME performance were optimized to achieve the highest extraction efficiency and the performance of the developed DLLME method was evaluated. Good linearity was observed over the range assayed $(0.031-4.0 \mu \mathrm{g} / \mathrm{mL})$ with regression coefficients greater than 0.9989 for all tocopherols and tocotrienols. Limits of detection and enrichment factors ranged from 0.01 to $0.11 \mu \mathrm{g} / \mathrm{mL}$ and 50 to 73 , respectively. Intra- and inter-day precision were lower than $8.9 \%$ and the recoveries were around $85.5-116.6 \%$ for all tocopherols and tocotrienols. The developed DLLME method was successfully applied to cereals: rice, barley, oat, wheat, corn and millet. This new sample preparation approach represents an inexpensive, rapid, simple and precise sample cleanup and concentration method for the determination of tocopherols and tocotrienols in cereals.
\end{abstract}

Keyword: Tocopherol; Tocotrienol; Analysis; HPLC; Microextraction; Cereal 